\title{
ON CONVERGENT SEQUENCES AND FIXED POINT THEOREMS IN D-METRIC SPACES
}

\author{
S. V. R. NAIDU, K. P. R. RAO, AND N. SRINIVASA RAO \\ Received 24 March 2004 and in revised form 7 January 2005
}

Examples of complete $D$-metric spaces are given in which every convergent sequence has at most two limits and in which there are convergent sequences with exactly two limits. Also an example of a complete $D$-metric space having a convergent sequence with infinitely many limits is given and, using the example, several fixed point theorems in $D$-metric spaces are shown to be false. Modifications of some of these theorems and their generalizations are obtained either by imposing restrictions on the number of limits of certain convergent sequences in the space or by assuming the sequential continuity of the $D$-metric in any two variables and the theorems so obtained are illustrated by means of examples.

\section{Introduction}

In this paper, we give examples (Examples 3.1 and 3.2) of complete $D$-metric spaces in which every convergent sequence has at most two limits and in which there are convergent sequences having exactly two limits. We also provide a simple example (Example 3.3) of a complete $D$-metric space in which there is a convergent sequence, which converges to every element of the space. Using the last one, we show that several fixed point theorems in $D$-metric spaces are false. In particular, we observe that Dhage [2, Theorem 2.1], Ahmad et al. [1, Theorem 4.2], and Dhage et al. [3, Theorem 3.1] are false and we modify them. We provide examples to illustrate these modifications. Further, we obtain generalizations of these modifications.

\section{Definitions}

Definition 2.1 (see [2]). Let $X$ be a nonempty set. A function $\rho: X \times X \times X \rightarrow[0, \infty)$ is called a $D$-metric on $X$ if

(i) $\rho(x, y, z)=0$ if and only if $x=y=z$ (coincidence);

(ii) $\rho(x, y, z)=\rho(p(x, y, z))$ for all $x, y, z \in X$ and for each permutation $p(x, y, z)$ of $x$, $y, z$ (symmetry);

(iii) $\rho(x, y, z) \leq \rho(x, y, a)+\rho(x, a, z)+\rho(a, y, z)$ for all $x, y, z, a \in X$ (tetrahedral inequality). 
If $X$ is a nonempty set and $\rho$ is a $D$-metric on $X$, then the ordered pair $(X, \rho)$ is called a $D$-metric space. When the $D$-metric $\rho$ is understood, $X$ itself is called a $D$-metric space. Definition 2.2 (see [2]). A sequence $\left\{x_{n}\right\}$ in a $D$-metric space $(X, \rho)$ is said to be convergent (or $\rho$-convergent) if there exists an element $x$ of $X$ with the following property. Given that $\varepsilon>0$, there exists an $N \in \mathbb{N}$ such that $\rho\left(x_{m}, x_{n}, x\right)<\varepsilon$ for all $m, n \geq N$. In such a case, $\left\{x_{n}\right\}$ is said to converge to $x$ and $x$ is said to be a limit of $\left\{x_{n}\right\}$.

Definition 2.3 (see [2]). A sequence $\left\{x_{n}\right\}$ in a $D$-metric space $(X, \rho)$ is said to be a Cauchy (or $\rho$-Cauchy) sequence if given that $\varepsilon>0$, there exists an $N \in \mathbb{N}$ such that $\rho\left(x_{m}, x_{n}, x_{p}\right)<\varepsilon$ for all $m, n, p \geq N$.

Definition 2.4 (see [2]). A $D$-metric space $X$ is said to be complete if every $\rho$-Cauchy sequence in $X$ converges to a point $x$ in $X$.

Definition 2.5 (see [2]). A $D$-metric space $(X, \rho)$ is said to be bounded if there exists a positive real number $M$ such that $\rho(x, y, z) \leq M$ for all $x, y, z \in X$. In such a case, $M$ is said to be a $D$-bound for the $D$-metric $\rho$.

Definition 2.6 (see [5]). A subset $E$ of a $D$-metric space $(X, \rho)$ is said to be $\rho$-bounded if there exists a positive real number $M$ such that $\rho(x, y, z) \leq M$ for all $x, y, z \in E$.

Definition 2.7 (see [6]). A pair $\left(f_{1}, f_{2}\right)$ of self-maps on an arbitrary set $E$ is said to be weakly compatible, partially commuting, or coincidentally commuting if $\left(f_{1} \circ f_{2}\right) u=$ $\left(f_{2} \circ f_{1}\right) u$ whenever $u \in E$ and $f_{1} u=f_{2} u$.

Remark 2.8. The definition of $\rho$-Cauchy sequence as given by Dhage [2] appears to be slightly different from Definition 2.3 but it is actually equivalent to it. It can be shown that in a $D$-metric space every convergent sequence is a Cauchy sequence.

\section{Examples and theorems}

Throughout this section, unless otherwise stated, $\mathbb{R}$ is the set of all real numbers, $\mathbb{R}^{+}$ is the set of all nonnegative real numbers, $\mathbb{N}$ is the set of all positive integers and for a monotonically increasing function $\varphi: \mathbb{R}^{+} \rightarrow \mathbb{R}^{+}$and $t \in \mathbb{R}^{+}, \varphi(t+)$ stands for the righthand limit of $\varphi$ at $t$.

We now give examples of complete $D$-metric spaces in which every convergent sequence has at most two limits and in which there are convergent sequences having exactly two limits.

Example 3.1. Let $X=\{0,1,1 / 2,1 / 3, \ldots\}$. Define $\rho: X \times X \times X \rightarrow \mathbb{R}^{+}$as follows:

$$
\rho(x, y, z)= \begin{cases}|x-y|+|y-z|+|z-x| & \text { if } x, y, z \in X \backslash\{1\}, \\ 0 & \text { if } x=y=z=1, \\ \min \{\max \{x, y\}, & \text { if both } 0 \text { and } 1 \text { occur among } x, y, z, \text { or } \\ \max \{y, z\}, \max \{z, x\}\} & \text { if } 1 \text { occurs exactly twice among } x, y, z, \\ & \text { if } 1 \text { occurs exactly once and } 0 \\ & \text { does not occur among } x, y, z .\end{cases}
$$


Then $(X, \rho)$ is a complete $D$-metric space. Further $\{1 / n\}$ converges to both 0 and 1 . If a sequence $\left\{x_{n}\right\}$ in $X$ converges to an element $x_{0} \in X \backslash\{0,1\}$, then $x_{0}$ is the unique limit of $\left\{x_{n}\right\}$. In particular, a convergent sequence in $X$ has at most two limits.

Proof. Clearly $\rho$ is symmetric in all the three variables $x, y, z$ and $\rho(x, y, z)=0 \Leftrightarrow x=y=$ $z$. Let $x, y, z, u \in X$.

Case 1. $x, y, z \in X \backslash\{1\}$.

If $u \in X \backslash\{1\}$, then

$$
\begin{aligned}
\rho(x, y, z)= & |x-y|+|y-z|+|z-x| \\
\leq & |u-y|+|y-z|+|z-u| \\
& +|x-u|+|u-z|+|z-x| \\
& +|x-y|+|y-u|+|u-x| \\
= & \rho(u, y, z)+\rho(x, u, z)+\rho(x, y, u) .
\end{aligned}
$$

If $u=1$, then

$$
\begin{aligned}
\rho(x, y, z) & =|x-y|+|y-z|+|z-x| \\
& \leq \max \{y, z\}+\max \{z, x\}+\max \{x, y\} \\
& \leq \rho(u, y, z)+\rho(x, u, z)+\rho(x, y, u)
\end{aligned}
$$

since $\rho(u, y, z)=\rho(1, y, z)=1$ or $\max \{y, z\}$, and so forth.

Case 2. 1 occurs among $x, y, z$.

Subcase 1. $x=y=z=1$.

Then

$$
\rho(x, y, z)=0 \leq \rho(u, y, z)+\rho(x, u, z)+\rho(x, y, u) .
$$

Subcase 2. 1 occurs exactly twice among $x, y, z$.

Without loss of generality, we may assume that $x=y=1$. Then $z \in X \backslash\{1\}$.

If $u=1$, then

$$
\begin{aligned}
\rho(x, y, z) & =\rho(1,1, z)=\rho(u, y, z) \\
& \leq \rho(u, y, z)+\rho(x, u, z)+\rho(x, y, u) .
\end{aligned}
$$

If $u \neq 1$, then

$$
\begin{aligned}
\rho(x, y, z) & =\rho(1,1, z)=1=\rho(1,1, u)=\rho(x, y, u) \\
& \leq \rho(u, y, z)+\rho(x, u, z)+\rho(x, y, u) .
\end{aligned}
$$

Subcase 3. 1 occurs exactly once among $x, y, z$.

Without loss of generality, we may assume that $x=1$. Then $y, z \in X \backslash\{1\}$. We may assume that $z \leq y$. 
If $u=1$, then

$$
\begin{aligned}
\rho(x, y, z) & =\rho(1, y, z)=\rho(u, y, z) \\
& \leq \rho(u, y, z)+\rho(x, u, z)+\rho(x, y, u) .
\end{aligned}
$$

If $z=0$, then

$$
\rho(x, y, z)=\rho(1, y, 0)=1=\rho(1, u, 0)=\rho(x, u, z),
$$

and hence

$$
\rho(x, y, z) \leq \rho(u, y, z)+\rho(x, u, z)+\rho(x, y, u) .
$$

If $u \neq 1$ and $z \neq 0$, then

$$
\begin{aligned}
\rho(x, y, z) & =\rho(1, y, z)=\max \{y, z\}=y \\
& \leq \max \{u, y\} \leq \rho(1, y, u)=\rho(x, y, u) \\
& \leq \rho(u, y, z)+\rho(x, u, z)+\rho(x, y, u) .
\end{aligned}
$$

Thus for all $x, y, z, u \in X$, we have

$$
\rho(x, y, z) \leq \rho(u, y, z)+\rho(x, u, z)+\rho(x, y, u) .
$$

Hence $\rho$ is a $D$-metric on $X$.

We are going to show that $(X, \rho)$ is complete. Let $\left\{x_{n}\right\}$ be a Cauchy sequence in $X$.

Case 1. There exists $N \in \mathbb{N}$ such that $x_{n}=x_{N}$ for all $n \geq N$.

In this case, evidently, $\left\{x_{n}\right\}$ converges to $x_{N}$.

Case 2. There does not exist $N \in \mathbb{N}$ such that $x_{n}=x_{N}$ for all $n \geq N$.

In this case, given $N \in \mathbb{N}$, there exist $i, j \in \mathbb{N}$ such that $i>j>N$ and $x_{i} \neq x_{j}$.

Subcase 1. There exists $\alpha \in X$ such that $x_{n}=\alpha$ for infinitely many $n$. Then given $N \in \mathbb{N}$, there exist $i, j, k \in \mathbb{N}$ such that $k>j>i>N, x_{i}=x_{j}=\alpha$, and $x_{k} \neq \alpha$. We have $\rho\left(x_{i}, x_{j}, x_{k}\right)=$ 1 if $\alpha=1$. Since $\left\{x_{n}\right\}$ is a Cauchy sequence, it follows that $\alpha \neq 1$. Hence, there exists $N_{0} \in$ $\mathbb{N}$ such that $x_{n} \in X \backslash\{1\}$ for all $n \geq N_{0}$. Hence, $\rho\left(x_{i}, x_{j}, x_{k}\right)=\left|x_{i}-x_{j}\right|+\left|x_{j}-x_{k}\right|+\left|x_{k}-x_{i}\right|$ for all $i, j, k \geq N_{0}$. Since $\left\{x_{n}\right\}$ is a Cauchy, $\rho\left(x_{i}, x_{j}, x_{k}\right) \rightarrow 0$ as $i, j, k \rightarrow \infty$. Since $x_{n}=\alpha$ for infinitely many $n$, given $i \in \mathbb{N}$, there exist $j, k \in \mathbb{N}$ such that $k>j>i$ and $x_{j}=x_{k}=\alpha$. Hence given an integer $i \geq N_{0}$, there exist $j, k \in \mathbb{N}$ such that $k>j>i$ and $2\left|x_{i}-\alpha\right|=\rho\left(x_{i}, x_{j}, x_{k}\right)$. Hence $\left|x_{i}-\alpha\right| \rightarrow 0$ as $i \rightarrow \infty$. Hence for $n, m \geq N_{0}, \rho\left(x_{n}, x_{m}, \alpha\right)=\left|x_{n}-x_{m}\right|+\left|x_{m}-\alpha\right|+$ $\left|\alpha-x_{n}\right| \rightarrow 0$ as $n, m \rightarrow \infty$. Hence $\left\{x_{n}\right\}$ converges to $\alpha$.

Subcase 2. For any $\alpha \in X, x_{n}=\alpha$ for only finitely many $n$.

Let $\varepsilon$ be a positive real number. Choose a positive integer $M$ such that $(1 / M)<\varepsilon$. Let $N_{1}=\sup \left\{n \in \mathbb{N}: x_{n} \in\{1,1 / 2,1 / 3, \ldots, 1 / M\}\right\}$, where we adopt the convention that the supremum of the empty subset of $\mathbb{R}$ is zero. Since for any $k \in \mathbb{N}, 1 / k \in X$ and $x_{n}=1 / k$ for only finitely many $n$, it follows that $N_{1}$ is a nonnegative integer. Let $N=N_{1}+1$. Then 
$0 \leq x_{n}<1 / M<\varepsilon$ for all $n \geq N$ since $X=\{0\} \cup\{1 / k: k \in \mathbb{N}\}$. Hence $\left\{x_{n}\right\}$ converges to 0 in the usual sense.

For $n, m \geq N$, we have

$$
\begin{aligned}
\rho\left(0, x_{n}, x_{m}\right) & =\left|0-x_{n}\right|+\left|x_{n}-x_{m}\right|+\left|x_{m}-0\right| \\
& \leq 2\left|x_{n}\right|+2\left|x_{m}\right| \\
& \longrightarrow 0 \quad \text { as } n, m \longrightarrow \infty .
\end{aligned}
$$

Hence $\left\{x_{n}\right\}$ converges to 0 with respect to $\rho$. Thus in any case, $\left\{x_{n}\right\}$ is convergent in $X$ with respect to $\rho$. Hence $(X, \rho)$ is complete.

Evidently $\{1 / n\}$ converges to both 0 and 1 .

Let $\left\{x_{n}\right\}$ be a sequence in $X$ such that it converges to an element $x_{0}$ of $X \backslash\{0,1\}$. Since $\rho\left(1,1, x_{0}\right)=1$ and $\rho\left(x_{n}, x_{m}, x_{0}\right) \rightarrow 0$ as $n, m \rightarrow \infty$, it follows that there exists $N_{0} \in \mathbb{N}$ such that $x_{n} \in X \backslash\{1\}$ for all $n \geq N_{0}$. For $n, m \geq N_{0}$, we have

$$
\rho\left(x_{n}, x_{m}, x_{0}\right)=\left|x_{n}-x_{m}\right|+\left|x_{m}-x_{0}\right|+\left|x_{0}-x_{n}\right| \text {. }
$$

Since $\rho\left(x_{n}, x_{m}, x_{0}\right) \rightarrow 0$ as $n, m \rightarrow \infty$, it follows that $\left|x_{n}-x_{0}\right| \rightarrow 0$ as $n \rightarrow \infty$. Since $x_{0}$ is positive, it follows that there exists $N_{1} \in \mathbb{N}$ such that $x_{n}>0$ for all $n \geq N_{1}$. For $m, n \geq$ $\max \left\{N_{0}, N_{1}\right\}$, we have

$$
\begin{aligned}
\rho\left(1, x_{n}, x_{m}\right) & =\min \left\{\max \left\{1, x_{n}\right\}, \max \left\{x_{n}, x_{m}\right\}, \max \left\{x_{m}, 1\right\}\right\}=\max \left\{x_{n}, x_{m}\right\} \\
& \longrightarrow x_{0} \text { as } n, m \longrightarrow \infty .
\end{aligned}
$$

Hence $\left\{x_{n}\right\}$ does not converge to 1 . Let $y \in X \backslash\{1\}$. For $m, n \geq N_{0}$, we have

$$
\begin{aligned}
\rho\left(y, x_{n}, x_{m}\right) & =\left|y-x_{n}\right|+\left|x_{n}-x_{m}\right|+\left|x_{m}-y\right| \\
& \longrightarrow 2\left|y-x_{0}\right| \quad \text { as } n, m \longrightarrow \infty, \\
2\left|y-x_{0}\right| & =0 \Longleftrightarrow y=x_{0} .
\end{aligned}
$$

Hence $\left\{x_{n}\right\}$ does not converge to $y$ for any $y \in X \backslash\left\{1, x_{0}\right\}$. Hence $x_{0}$ is the only limit of $\left\{x_{n}\right\}$.

Example 3.2. Let $X=\{0,1,1 / 2,1 / 3, \ldots\}$. Define $\rho: X \times X \times X \rightarrow \mathbb{R}^{+}$as

$$
\rho(x, y, z)= \begin{cases}|x-y|+|y-z|+|z-x| & \text { if } x, y, z \in X \backslash\{1\}, \\ 0 & \text { if } x=y=z=1, \\ 1 & \text { if both } 0 \text { and } 1 \text { occur among } x, y, z, \\ \max \{y, z\}, \max \{z, x\}\} & \text { if } 1 \text { occurs exactly once and } \\ & 0 \text { does not occur among } x, y, z, \\ 2 \min \{x, y, z\} & \text { if } 1 \text { occurs exactly twice among } x, y, z \text { and } \\ & 0 \text { does not occur among } x, y, z .\end{cases}
$$

Then $(X, \rho)$ is a complete $D$-metric space. Further $\{1 / n\}$ converges to both 0 and 1 . 


\section{On convergent sequences in $D$-metric spaces}

If a sequence $\left\{x_{n}\right\}$ in $X$ converges to an element $x_{0} \in X \backslash\{0,1\}$, then $x_{0}$ is the unique limit of $\left\{x_{n}\right\}$. In particular, a convergent sequence in $X$ has at most two limits.

Proof. Clearly, $\rho$ is symmetric in all the three variables $x, y, z$ and $\rho(x, y, z)=0 \Leftrightarrow x=y=$ $z$. Let $x, y, z, u \in X$.

Case 1. $x, y, z \in X \backslash\{1\}$.

If $u \in X \backslash\{1\}$, then

$$
\begin{aligned}
\rho(x, y, z)= & |x-y|+|y-z|+|z-x| \\
\leq & |u-y|+|y-z|+|z-u| \\
& +|x-u|+|u-z|+|z-x| \\
& +|x-y|+|y-u|+|u-x| \\
= & \rho(u, y, z)+\rho(x, u, z)+\rho(x, y, u) .
\end{aligned}
$$

If $u=1$, then

$$
\begin{aligned}
\rho(x, y, z) & =|x-y|+|y-z|+|z-x| \\
& \leq \max \{y, z\}+\max \{z, x\}+\max \{x, y\} \\
& \leq \rho(u, y, z)+\rho(x, u, z)+\rho(x, y, u)
\end{aligned}
$$

since $\rho(u, y, z)=\rho(1, y, z)=1$ or $2 \max \{y, z\}$, and so forth.

Case 2. 1 occurs among $x, y, z$.

Subcase 1. $x=y=z=1$.

Then

$$
\rho(x, y, z)=0 \leq \rho(u, y, z)+\rho(x, u, z)+\rho(x, y, u) .
$$

Subcase 2. 1 occurs exactly twice among $x, y, z$.

Without loss of generality, we may assume that $x=y=1$. Then $z \in X \backslash\{1\}$.

If $u=1$, then

$$
\begin{aligned}
\rho(x, y, z) & =\rho(1, y, z)=\rho(u, y, z) \\
& \leq \rho(u, y, z)+\rho(x, u, z)+\rho(x, y, u) .
\end{aligned}
$$

If $z=0$, then

$$
\begin{aligned}
\rho(x, y, z) & =\rho(1,1,0)=1=\rho(u, 1,0)=\rho(u, y, z) \\
& \leq \rho(u, y, z)+\rho(x, u, z)+\rho(x, y, u) .
\end{aligned}
$$

If $z \neq 0$ and $u \neq 1$, then

$$
\begin{aligned}
\rho(x, y, z) & =\rho(1,1, z)=2 z \\
& \leq 2 \max \{u, z\} \leq \rho(u, 1, z)=\rho(u, y, z) \\
& \leq \rho(u, y, z)+\rho(x, u, z)+\rho(x, y, u) .
\end{aligned}
$$


Subcase 3. 1 occurs exactly once among $x, y, z$.

Without loss of generality, we may assume that $x=1$. Then $y, z \in X \backslash\{1\}$.

We may assume that $z \leq y$.

If $u=1$, then

$$
\rho(x, y, z)=\rho(1, y, z)=\rho(u, y, z) \leq \rho(u, y, z)+\rho(x, u, z)+\rho(x, y, u) .
$$

If $z=0$, then

$$
\rho(x, y, z)=\rho(1, y, 0)=1=\rho(1, u, 0)=\rho(x, u, z),
$$

and hence

$$
\rho(x, y, z) \leq \rho(u, y, z)+\rho(x, u, z)+\rho(x, y, u) .
$$

If $u \neq 1$ and $z \neq 0$, then

$$
\begin{aligned}
\rho(x, y, z) & =\rho(1, y, z)=2 \max \{y, z\} \\
& =2 y \leq 2 \max \{u, y\} \leq \rho(1, y, u) \\
& =\rho(x, y, u) \leq \rho(u, y, z)+\rho(x, u, z)+\rho(x, y, u) .
\end{aligned}
$$

Thus for all $x, y, z, u \in X$, we have

$$
\rho(x, y, z) \leq \rho(u, y, z)+\rho(x, u, z)+\rho(x, y, u) .
$$

Hence $\rho$ is a $D$-metric on $X$.

We are going to show that $(X, \rho)$ is complete. Let $\left\{x_{n}\right\}$ be a Cauchy sequence in $X$.

Case 1. $x_{n}=1$ for infinitely many $n$.

Given $i \in \mathbb{N}$, we can choose $j, k \in \mathbb{N}$ such that $k>j>i$ and $x_{k}=x_{j}=1$ so that

$$
\rho\left(x_{i}, x_{j}, x_{k}\right)=\rho\left(x_{i}, 1,1\right)= \begin{cases}0 & \text { if } x_{i}=1 \\ 1 & \text { if } x_{i}=0 \\ 2 x_{i} & \text { if } x_{i} \in X \backslash\{0,1\} .\end{cases}
$$

Since $\left\{x_{n}\right\}$ is a Cauchy sequence, $\rho\left(x_{p}, x_{q}, x_{r}\right) \rightarrow 0$ as $p, q, r \rightarrow \infty$.

Hence, $\rho\left(x_{i}, 1,1\right) \rightarrow 0$ as $i \rightarrow \infty$. Hence, $x_{n}=0$ for only finitely many $n$. Hence, there exists $N \in \mathbb{N}$ such that $x_{n} \neq 0$ for all $n \geq N$.

If $x_{n} \neq 1$ for infinitely many $n$, then $x_{n} \rightarrow 0$ in the usual sense as $n \rightarrow \infty$ among those $n$ for which $x_{n} \neq 1$.

For $m, n \geq N$, we have

$$
\rho\left(1, x_{m}, x_{n}\right)= \begin{cases}0 & \text { if } x_{m}=x_{n}=1, \\ 2 x_{m} & \text { if } x_{n}=1, x_{m} \neq 1, \\ 2 x_{n} & \text { if } x_{m}=1, x_{n} \neq 1, \\ 2 \max \left\{x_{m}, x_{n}\right\} & \text { if } x_{m} \neq 1, x_{n} \neq 1 .\end{cases}
$$

Hence, $\rho\left(1, x_{m}, x_{n}\right) \rightarrow 0$ as $m, n \rightarrow \infty$. Hence, $\left\{x_{n}\right\}$ converges to 1 with respect to $\rho$. 
Case 2. $x_{n}=1$ for only finitely many $n$ and there exists $\alpha \in X \backslash\{1\}$ such that $x_{n}=\alpha$ for infinitely many $n$.

Since $x_{n}=1$ for only finitely many $n$, there exists $N_{0} \in \mathbb{N}$ such that $x_{n} \in X \backslash\{1\}$ for all $n \geq N_{0}$. Hence, $\rho\left(x_{i}, x_{j}, x_{k}\right)=\left|x_{i}-x_{j}\right|+\left|x_{j}-x_{k}\right|+\left|x_{k}-x_{i}\right|$ for all $i, j, k \geq N_{0}$. Since $x_{n}=\alpha$ for infinitely many $n$, given $i \in \mathbb{N}$, there exist $j, k \in \mathbb{N}$ such that $k>j>i$ and $x_{j}=x_{k}=\alpha$.

Hence given an integer $i \geq N_{0}$, there exist $j, k \in \mathbb{N}$ such that $k>j>i$ and

$$
2\left|x_{i}-\alpha\right|=\rho\left(x_{i}, x_{j}, x_{k}\right) .
$$

Since $\left\{x_{n}\right\}$ is a Cauchy sequence, $\rho\left(x_{i}, x_{j}, x_{k}\right) \rightarrow 0$ as $i, j, k \rightarrow \infty$. Hence, $\left|x_{i}-\alpha\right| \rightarrow 0$ as $i \rightarrow \infty$. Hence for $n, m \geq N_{0}$,

$$
\begin{aligned}
\rho\left(x_{n}, x_{m}, \alpha\right) & =\left|x_{n}-x_{m}\right|+\left|x_{m}-\alpha\right|+\left|\alpha-x_{n}\right| \\
& \leq 2\left|x_{m}-\alpha\right|+2\left|x_{n}-\alpha\right| \\
& \longrightarrow 0 \text { as } m, n \longrightarrow \infty .
\end{aligned}
$$

Hence $\left\{x_{n}\right\}$ converges to $\alpha$.

Case 3. For any $\alpha \in X, x_{n}=\alpha$ for only finitely many $n$.

Let $\varepsilon$ be a positive real number. Choose a positive integer $M$ such that $(1 / M)<\varepsilon$.

Let $N_{1}=\sup \left\{n \in \mathbb{N}: x_{n} \in\{1,1 / 2,1 / 3, \ldots, 1 / M\}\right\}$, where we adopt the convention that the supremum of the empty subset of $\mathbb{R}$ is zero. Since for any $k \in \mathbb{N}, 1 / k \in X$ and $x_{n}=1 / k$ for only finitely many $n$, it follows that $N_{1}$ is a nonnegative integer.

Let $N=N_{1}+1$. Since $X=\{0,1,1 / 2,1 / 3, \ldots\}$, it follows that $0 \leq x_{n}<1 / M<\varepsilon$ for all $n \geq N$.

Hence $\left\{x_{n}\right\}$ converges to 0 in the usual sense.

For $n, m \geq N$, we have

$$
\begin{aligned}
\rho\left(0, x_{n}, x_{m}\right) & =\left|0-x_{n}\right|+\left|x_{n}-x_{m}\right|+\left|x_{m}-0\right| \\
& \leq 2\left|x_{n}\right|+2\left|x_{m}\right| \\
& \longrightarrow 0 \text { as } n, m \longrightarrow \infty .
\end{aligned}
$$

Hence $\left\{x_{n}\right\}$ converges to 0 with respect to $\rho$. Thus in any case, $\left\{x_{n}\right\}$ is convergent in $X$ with respect to $\rho$. Hence $(X, \rho)$ is complete.

Evidently, $\{1 / n\}$ converges to both 0 and 1 .

If a sequence $\left\{x_{n}\right\}$ in $X$ converges to an element $x_{0} \in X \backslash\{0,1\}$, then it can be shown as in Example 3.1 that $x_{0}$ is a unique limit of $\left\{x_{n}\right\}$.

We now give a simple example of a complete $D$-metric space having a convergent sequence, which converges to every element of the space.

Example 3.3. Let $X=\left\{1 / 2^{n}: n \in \mathbb{N}\right\}$. Define $\rho: X \times X \times X \rightarrow \mathbb{R}^{+}$as follows:

$$
\rho(x, y, z)= \begin{cases}0 & \text { if } x=y=z, \\ \min \{\max \{x, y\}, \max \{y, z\}, \max \{z, x\}\} & \text { otherwise. }\end{cases}
$$


Then $(X, \rho)$ is a complete $D$-metric space. Further $\left\{1 / 2^{n}\right\}_{n=1}^{\infty}$ is a $\rho$-Cauchy sequence and converges with respect to $\rho$ to $x$ for all $x \in X$.

Proof. We are going to prove that $\rho$ is a $D$-metric on $X$. Clearly $\rho$ is symmetric in all the three variables and $\rho(x, y, z)=0 \Leftrightarrow x=y=z$. We note that $\rho(x, y, z) \leq 1 / 2$ for all $x, y, z \in X$. Let $x, y, z \in X$ and at least let two among $x, y, z$ be distinct. We may assume that $x \geq y \geq z$. Then $\rho(x, y, z)=y$.

Let $u \in X$. When $u \geq y, \rho(u, y, z)=y$; and when $u<y, \rho(x, y, u)=y$. Hence $\rho(x, y, z) \leq$ $\rho(u, y, z)+\rho(x, u, z)+\rho(x, y, u)$. Hence $\rho$ is a $D$-metric on $X$.

We are going to show that $(X, \rho)$ is complete. Let $\left\{x_{n}\right\}$ be a Cauchy sequence in $X$.

Case 1. There exists $N \in \mathbb{N}$ such that $x_{n}=x_{N}$ for all $n \geq N$.

In this case, evidently $\left\{x_{n}\right\}$ converges to $x_{N}$.

Case 2. Given $N \in \mathbb{N}$, there exist $i, j \in \mathbb{N}$ such that $i>N, j>N$, and $x_{i} \neq x_{j}$.

Claim 3.4. $x_{n} \rightarrow 0$ as $n \rightarrow \infty$ in the usual sense.

Suppose not. Then there exists a positive real number $\varepsilon$ such that $x_{n} \geq \varepsilon$ for infinitely many $n \in \mathbb{N}$. Given $N \in \mathbb{N}$, we can choose $i, j, k \in \mathbb{N}$ such that $k>j>i>N, x_{i} \geq \varepsilon, x_{j} \geq \varepsilon$, and $x_{k} \neq x_{j}$. Then

$$
\rho\left(x_{i}, x_{j}, x_{k}\right)=\min \left\{\max \left\{x_{i}, x_{j}\right\}, \max \left\{x_{j}, x_{k}\right\}, \max \left\{x_{k}, x_{i}\right\}\right\} \geq \varepsilon .
$$

This is a contradiction, since $\left\{x_{n}\right\}$ is a Cauchy sequence.

For $m, n \in \mathbb{N}$ and $x \in X$, we have

$$
\begin{aligned}
\rho\left(x, x_{m}, x_{n}\right) & \leq \min \left\{\max \left\{x, x_{m}\right\}, \max \left\{x_{m}, x_{n}\right\}, \max \left\{x_{n}, x\right\}\right\} \\
& \leq \max \left\{x_{m}, x_{n}\right\} \\
& \longrightarrow 0 \text { as } m, n \longrightarrow \infty .
\end{aligned}
$$

Hence $\left\{x_{n}\right\}$ converges to $x$ for any $x \in X$ with respect to the $D$-metric $\rho$.

Hence $(X, \rho)$ is a complete $D$-metric space.

Dhage [2] proved the following theorem.

Theorem 3.5 (see [2, Theorem 2.1]). Let $f$ be a self-map of a complete and bounded

$D$-metric space $X$ such that

$$
\rho(f x, f y, f z) \leq \alpha \rho(x, y, z)
$$

for all $x, y, z \in X$, and some $\alpha \in[0,1)$. Then $f$ has a unique fixed point.

Remark 3.6. Example 3.7 shows that the existence part of fixed point of Theorem 3.5 is false.

Example 3.7. Let $(X, \rho)$ be as in Example 3.3. Define $f: X \rightarrow X$ as $f x=x / 2$ for all $x \in$ $X$. Then $\rho(f x, f y, f z) \leq(1 / 2) \rho(x, y, z)$ for all $x, y, z \in X$ but $f$ has no fixed point in $X$. Further for all $x \in X,\left\{f^{n} x\right\}$ converges to every element of $X$, and hence has infinitely many limits. 
1978 On convergent sequences in $D$-metric spaces

Proof. Let $x, y, z \in X$

Case 1. $x=y=z$.

Then

$$
\rho(f x, f y, f z)=\rho(f x, f x, f x)=0=\frac{1}{2} \rho(x, x, x)=\frac{1}{2} \rho(x, y, z) .
$$

Case 2. At least two among $x, y, z$ are distinct.

We may assume that $x \geq y \geq z$. Then

$$
\rho(f x, f y, f z)=\rho\left(\frac{x}{2}, \frac{y}{2}, \frac{z}{2}\right)=\frac{y}{2}=\frac{1}{2} \rho(x, y, z) .
$$

Thus

$$
\rho(f x, f y, f z) \leq \frac{1}{2} \rho(x, y, z) \quad \forall x, y, z \in X .
$$

Evidently $f$ has no fixed point and for any $x \in X,\left\{f^{n} x\right\}$ in $X$ converges to every point of $X$.

The following is a modification of Dhage [2, Theorem 2.1].

Theorem 3.8. Let $(X, \rho)$ be a bounded complete D-metric space and let $f: X \rightarrow X$ be such that

$$
\rho(f x, f y, f z) \leq \alpha \rho(x, y, z)
$$

for all $x, y, z \in X$, and some $\alpha \in[0,1)$. Then for any $x \in X,\left\{f^{n} x\right\}$ is a D-Cauchy sequence. If there exists $x_{0} \in X$ such that $\left\{f^{n} x_{0}\right\}$ has only a finite number of limits, then fhas a unique fixed point.

We now illustrate Theorem 3.8 using the complete $D$-metric spaces given in Examples 3.1 and 3.2.

Example 3.9. Let $(X, \rho)$ be as in Example 3.1. Define $f: X \rightarrow X$ as

$$
f x= \begin{cases}0 & \text { if } x=1 \\ \frac{x}{3} & \text { otherwise }\end{cases}
$$

Then $\rho(f x, f y, f z) \leq(2 / 3) \rho(x, y, z)$ for all $x, y, z \in X$. Evidently, 0 is the unique fixed point of $f$.

Proof. Let $x, y, z \in X$. In view of the symmetry of $\rho$, it is enough to consider the following five cases.

Case 1. $x=y=z=1$.

Then

$$
\rho(f x, f y, f z)=\rho(f 1, f 1, f 1)=\rho(0,0,0)=0 \leq \frac{2}{3} \rho(x, y, z) .
$$


Case 2. $x=y=1$ and $z \neq 1$.

Then

$$
\begin{aligned}
\rho(f x, f y, f z) & =\rho(f 1, f 1, f z) \\
& =\rho\left(0,0, \frac{z}{3}\right)=\frac{2 z}{3}<\frac{2}{3}(1)=\frac{2}{3} \rho(x, y, z) .
\end{aligned}
$$

Case 3. $x=1, y=0$ and $z \neq 1$.

Then

$$
\begin{aligned}
\rho(f x, f y, f z) & =\rho(f 1, f 0, f z) \\
& =\rho\left(0,0, \frac{z}{3}\right)=\frac{2 z}{3}<\frac{2}{3}(1)=\frac{2}{3} \rho(x, y, z) .
\end{aligned}
$$

Case 4. $x=1$ and $0<z \leq y<1$.

Then

$$
\begin{aligned}
\rho(f x, f y, f z) & =\rho(f 1, f y, f z) \\
& =\rho\left(0, \frac{y}{3}, \frac{z}{3}\right)=\frac{2 y}{3}=\frac{2}{3} \rho(x, y, z) .
\end{aligned}
$$

Case 5. $z \leq y \leq x<1$.

Then

$$
\begin{aligned}
\rho(f x, f y, f z) & =\rho\left(\frac{x}{3}, \frac{y}{3}, \frac{z}{3}\right) \\
& =\left|\frac{x}{3}-\frac{y}{3}\right|+\left|\frac{y}{3}-\frac{z}{3}\right|+\left|\frac{z}{3}-\frac{x}{3}\right| \\
& =\frac{2}{3}(x-z) \leq \frac{2}{3} 2(x-z)=\frac{2}{3} \rho(x, y, z) .
\end{aligned}
$$

Thus $\rho(f x, f y, f z) \leq(2 / 3) \rho(x, y, z)$ for all $x, y, z \in X$.

Example 3.10. Let $(X, \rho)$ be as in Example 3.2. Define $f: X \rightarrow X$ and $g: X \rightarrow X$ as follows:

$$
\begin{aligned}
& f x= \begin{cases}0 & \text { if } x=1 \\
\frac{x}{3} & \text { otherwise }\end{cases} \\
& g x= \begin{cases}1 & \text { if } x \in\{0,1\} \\
\frac{x}{3} & \text { otherwise }\end{cases}
\end{aligned}
$$

It can be verified that

$$
\begin{aligned}
\rho(f x, f y, f z) & \leq \frac{1}{3} \rho(x, y, z), \\
\rho(g x, g y, g z) & \leq \frac{1}{3} \rho(x, y, z)
\end{aligned}
$$

for all $x, y, z \in X$. 
For any $x \in X \backslash\{0,1\}$, the sequences $\left\{f^{n} x\right\}$ and $\left\{g^{n} x\right\}$ are the same and converge to 0 and 1 only. While 0 is the fixed point of $f, 1$ is the fixed point of $g$ in $X$.

Theorem 3.8 is a corollary of the following theorem.

Theorem 3.11. Let $(X, \rho)$ be a D-metric space, let $f$ be a self-map on $X$, and let $\varphi: \mathbb{R}^{+} \rightarrow \mathbb{R}^{+}$ be a monotonically increasing map such that $\varphi^{n}(t) \rightarrow 0$ as $n \rightarrow \infty$ for each $t \in \mathbb{R}^{+}$. Suppose that

$$
\rho(f x, f y, f z) \leq \varphi(\rho(x, y, z))
$$

for all $x, y, z \in X$ and that there exists $x_{0} \in X$ such that $\left\{\rho\left(x_{0}, f^{i} x_{0}, f^{j} x_{0}\right): i, j \in\{0\} \cup \mathbb{N}\right\}$ is bounded. Then $\left\{f^{n} x_{0}\right\}$ is a $\rho$-Cauchy sequence. Suppose that $\left\{f^{n} x_{0}\right\}$ is convergent and has only a finite number of limits. Then $f$ has a unique fixed point in $X$, which is a limit of $\left\{f^{n} x_{0}\right\}$.

Proof. Let $x_{n}=f^{n} x_{0}$ for all $n \in \mathbb{N}$.

For nonnegative integers $r$ and $s$, and $n \in \mathbb{N}$, we have

$$
\begin{array}{rrr}
\rho\left(x_{n}, x_{n+r}, x_{n+s}\right) & =\rho\left(f^{n} x_{0}, f^{n} x_{r}, f^{n} x_{s}\right) & \\
& \leq \varphi\left(\rho\left(f^{n-1} x_{0}, f^{n-1} x_{r}, f^{n-1} x_{s}\right)\right) & (\text { from }(3.49)) \\
& \leq \varphi\left(\varphi\left(\rho\left(f^{n-2} x_{0}, f^{n-2} x_{r}, f^{n-2} x_{s}\right)\right)\right) & (\text { from }(3.49) \text { and the monotonic } \\
& & \\
& & \\
& \leq \varphi^{n}\left(\rho\left(x_{0}, x_{r}, x_{s}\right)\right) .
\end{array}
$$

Since $\left\{\rho\left(x_{0}, f^{i} x_{0}, f^{j} x_{0}\right): i, j \in\{0\} \cup \mathbb{N}\right\}$ is bounded, there exists a positive real number $M$ such that $\rho\left(x_{0}, f^{i} x_{0}, f^{j} x_{0}\right) \leq M$ for all $i, j \in\{0\} \cup \mathbb{N}$. Hence from inequality (3.50) and the monotonic increasing nature of $\varphi$, we have $\rho\left(x_{n}, x_{n+r}, x_{n+s}\right) \leq \varphi^{n}(M)$. Since $\varphi^{n}(M) \rightarrow 0$ as $n \rightarrow \infty$, from the above inequality, it follows that $\left\{x_{n}\right\}$ is a $\rho$-Cauchy sequence.

Suppose now that $\left\{f^{n} x_{0}\right\}$ is convergent and has only a finite number of limits. Let $S$ denote the set of all limits of $\left\{f^{n} x_{0}\right\}$. Then $S$ is a nonempty finite subset of $X$. Since $\varphi$ is a nonnegative real-valued monotonically increasing function on $\mathbb{R}^{+}$and $\varphi^{n}(t) \rightarrow 0$ as $n \rightarrow \infty$, it follows that $\varphi(t)<t$ for all $t \in(0, \infty)$ and $\varphi(0)=0$. Let $z \in S$. Then

$$
\begin{aligned}
\rho\left(f^{n+1} x_{0}, f^{m+1} x_{0}, f z\right) & \leq \varphi\left(\rho\left(f^{n} x_{0}, f^{m} x_{0}, z\right)\right) \\
& \leq \rho\left(f^{n} x_{0}, f^{m} x_{0}, z\right) \\
& \longrightarrow 0 \text { as } m, n \longrightarrow \infty .
\end{aligned}
$$

Hence $\left\{f^{n} x_{0}\right\}$ converges to $f z$ also.

Hence, $f z \in S$. Thus $f(S) \subseteq S$. For any positive integer $m$, we have $f^{m}(S) \subseteq f^{m-1}(S)$, where $f^{0}=I$, the identity map on $X$.

Suppose that $m_{0}$ is a positive integer such that $f^{m_{0}-1}(S)$ has at least two elements. Let $\beta=\inf \left\{\rho(x, y, z): x, y, z \in f^{m_{0}-1}(S)\right.$ and at least two of $\mathrm{x}, \mathrm{y}, \mathrm{z}$ are different $\}$. Since $f^{m_{0}-1}(S)$ 
is a finite set having at least two elements, $\left\{\rho(x, y, z): x, y, z \in f^{m_{0}-1}(S)\right.$ and at least two of $x, y, z$ are different $\}$ is a nonempty finite subset of $(0, \infty)$. Hence, there exist $u, v, z \in$ $f^{m_{0}-1}(S)$ such that at least two of them are distinct and $\rho(u, v, z)=\beta$. We have $\rho(f u, f v, f z)$ $\leq \varphi(\rho(u, v, z))=\varphi(\beta)<\beta$, since $\beta$ is a positive real number and $\varphi(t)<t$ for all $t \in(0, \infty)$. Since $u, v, z \in f^{m_{0}-1}(S), f u, f v, f z \in f^{m_{0}}(S) \subseteq f^{m_{0}-1}(S)$. Hence from the definition of $\beta$, it follows that $f u=f v=f z$. Since $f^{m_{0}-1}(S)$ is finite, at least two of $u, v, z$ are distinct and $f u=f v=f z$, it follows that $f^{m_{0}}(S)$ is a proper subset of $f^{m_{0}-1}(S)$. Since $S$ is finite, from the above discussion, it follows that $f^{n_{0}-1}(S)$ is a singleton for some $n_{0} \in \mathbb{N}$.

Let $f^{n_{0}-1}(S)=\{w\}$. Since $f^{n_{0}}(S) \subseteq f^{n_{0}-1}(S)$, we must have $f w=w$.

Remark 3.12. Examples 3.9 and 3.10 illustrate Theorem 3.11.

Remark 3.13. Example 3.7 shows that Dhage and Rhoades [4, Theorem 2] and its corollary are false.

Ahmad et al. [1] proved the following theorem.

Theorem 3.14. Let $(X, \rho)$ be a complete D-metric space. Let $f$ and $S$ be commutative selfmaps on $X$ such that $f$ is injective, $S$ is surjective, and

$$
\rho(f x, f y, f z) \leq \alpha \rho(S x, S y, S z)
$$

for all $x, y, z \in X$, and some $\alpha \in[0,1)$. Then there exists a unique common fixed point of $f$ and $S$.

Remark 3.15. Example 3.7 shows that Theorem 3.14 is false since the map $f$ in the example is injective, the identity map $I$ is surjective, $f \circ I=I \circ f$, and inequality (3.52) is true for all $x, y, z$ in $X$ with $\alpha=1 / 2$ and $S=I$. Theorem 3.14 remains valid if its hypothesis is strengthened by imposing the additional condition that $(X, \rho)$ is bounded and that every $\rho$-Cauhy sequence in $f(X)$ is convergent in $S(X)$ and has a unique limit in $S(X)$.

The following is a generalization of the modification of Theorem 3.14 suggested above.

Theorem 3.16. Let $(X, \rho)$ be a bounded complete D-metric space, let $f$ and $S$ be self-maps on $X$. Let $\varphi: \mathbb{R}^{+} \rightarrow \mathbb{R}^{+}$be a monotonically increasing map such that $\varphi^{n}(t) \rightarrow 0$ as $n \rightarrow \infty$, for each $t \in(0, \infty)$. Suppose that $f$ and $S$ are partially commuting, $f(X) \subseteq S(X)$,

$$
\rho(f x, f y, f z) \leq \varphi(\rho(S x, S y, S z))
$$

for all $x, y, z$ in $X$ and that every $\rho$-Cauchy sequence in $f(X)$ is convergent in $S(X)$ and has a unique limit in $S(X)$. Then $f$ and $S$ have a unique common fixed point in $X$.

Proof. Let $x_{0} \in X$. Since $f(X) \subseteq S(X)$, there exists a sequence $\left\{x_{n}\right\}$ in $X$ such that $f x_{n}=$ $S x_{n+1}$ for $n=0,1,2, \ldots$

Let $y_{n}=f x_{n}$. Since $\varphi: \mathbb{R}^{+} \rightarrow \mathbb{R}^{+}$is monotonically increasing on $\mathbb{R}^{+}$and $\left\{\varphi^{n}(t)\right\} \rightarrow 0$ as $n \rightarrow \infty$ for all $t \in(0, \infty), \varphi(t)<t$ for all $t \in(0, \infty)$. Hence $\varphi(0+)=0$. 
For positive integers $m, n, p$, from inequality (3.53), we have

$$
\begin{aligned}
\rho\left(y_{n}, y_{m}, y_{p}\right) & =\rho\left(f x_{n}, f x_{m}, f x_{p}\right) \\
& \leq \varphi\left(\rho\left(S x_{n}, S x_{m}, S x_{p}\right)\right) \quad(\text { from }(3.53)) \\
& =\varphi\left(\rho\left(y_{n-1}, y_{m-1}, y_{p-1}\right)\right),
\end{aligned}
$$

that is, $\rho\left(y_{n}, y_{m}, y_{p}\right) \leq \varphi\left(\rho\left(y_{n-1}, y_{m-1}, y_{p-1}\right)\right)$. Hence for $n \in \mathbb{N}$ and nonnegative integers $i$ and $j$, from the monotonic increasing nature of $\varphi$, we have

$$
\rho\left(y_{n}, y_{n+i}, y_{n+j}\right) \leq \varphi^{n}\left(\rho\left(y_{0}, y_{i}, y_{j}\right)\right) \text {. }
$$

Let $M=\sup \left\{\rho\left(y_{0}, y_{i}, y j\right): i, j \in \mathbb{N} \cup\{0\}\right\}$. Since $(X, \rho)$ is bounded, $M<\infty$. Hence from inequality (3.55), we have

$$
\rho\left(y_{n}, y_{n+i}, y_{n+j}\right) \leq \varphi^{n}(M)
$$

for all $n \in \mathbb{N}$ and $i, j \in \mathbb{N} \cup\{0\}$.

Since $\varphi^{n}(M) \rightarrow 0$ as $n \rightarrow \infty$, from the above inequality, it follows that $\left\{y_{n}\right\}$ is a $\rho$ Cauchy sequence. Since $\left\{y_{n}\right\}=\left\{f x_{n}\right\} \subseteq f(X)$ and every $\rho$-Cauchy sequence in $f(X)$ is convergent in $S(X)$ and has a unique limit in $S(X)$, there exists a unique element $v$ of $S(X)$ such that $\left\{y_{n}\right\}$ converges to $v$. Since $v \in S(X)$, there exists $u \in X$ such that $S u=v$.

For positive integers $n, m$, we have

$$
\begin{aligned}
\rho\left(y_{n}, y_{m}, f u\right) & =\rho\left(f x_{n}, f x_{m}, f u\right) \\
& \leq \varphi\left(\rho\left(S x_{n}, S x_{m}, S u\right)\right) \\
& =\varphi\left(\rho\left(y_{n-1}, y_{m-1}, v\right)\right) .
\end{aligned}
$$

Since $\left\{y_{n}\right\}$ converges to $v$ and $\varphi(0+)=0$, from the above inequality, it follows that $\left\{y_{n}\right\}$ converges to $f u$ also. Since $v$ is the only limit of $\left\{y_{n}\right\}$ in $S(X)$ and $f u \in f(X) \subseteq S(X)$, we must have $f u=v$. Hence $f u=S u$. Since $f$ and $S$ are partially commuting and $f u=S u$, we have $f(f u)=S(f u)$.

On taking $x=y=u$ and $z=f u$, in inequality (3.53), we obtain

$$
\rho\left(f u, f u, f^{2} u\right) \leq \varphi\left(\rho\left(f u, f u, f^{2} u\right)\right) .
$$

Since $\varphi(t)<t$ for all $t \in(0, \infty)$, we must have $\rho\left(f u, f u, f^{2} u\right)=0$. Hence $f^{2} u=f u$. Hence $S(f u)=f u$. Thus $f u$ is a common fixed point of $f$ and $S$.

From inequality (3.53), it is evident that $f$ and $S$ cannot have more than one common fixed point.

Remark 3.17. The following example illustrates Theorem 3.16.

Example 3.18. Let $X=\{0,1,1 / 2,1 / 3, \ldots\}$. Define $\rho: X \times X \times X \rightarrow \mathbb{R}^{+}$as

$$
\rho(x, y, z)=|x-y|+|y-z|+|z-x|, \quad \forall x, y, z \in X .
$$

Then $(X, \rho)$ is a bounded complete $D$-metric space in which every convergent sequence has a unique limit. 
Define $f: X \rightarrow X$ as $f(x)=(1 / 2) x$ for all $x \in X$. Then $\rho(f x, f y, f z) \leq(1 / 2) \rho(x, y, z)$ for all $x, y, z \in X$. Clearly $f$ has a unique fixed point in $X$, namely, 0 .

Proposition 3.19. Let $(X, \rho)$ be a $D$-metric space and let $\rho$ be sequentially continuous in one variable. Then every $\rho$-convergent sequence in $X$ has a unique limit.

Proof. Let $\left\{x_{n}\right\}$ be a $\rho$-convergent sequence in $X$. Let $x, y$ be limits of $\left\{x_{n}\right\}$. Since $\rho$ is sequentially continuous in one variable, $\left\{\rho\left(x_{n}, x, x\right)\right\}$ converges to $\rho(x, x, x)$ as well as $\rho(y, x, x)$. Since a convergent sequence of real numbers has a unique limit, we must have $\rho(y, x, x)=\rho(x, x, x)$. Hence $\rho(y, x, x)=0$. Hence $y=x$. Hence every $\rho$-convergent sequence in $X$ has a unique limit.

Remark 3.20. Since each of the D-metric spaces given in Examples 3.1, 3.2, and 3.3 contains a convergent sequence with several limits, the $D$-metric in any of these spaces is not sequentially continuous even in a single variable. It was observed by Naidu et al. [7] that a $D$-metric need not be sequentially continuous even in a single variable even when $D$-metric convergence defines a metrizable topology.

Notation 3.21. For a subset $E$ of a $D$-metric space $(X, \rho), \bar{E}$ denotes the set of all $x \in X$ such that there is a sequence in $E$ which converges to $x$ with respect to $\rho$.

Dhage et al. [3] proved the following theorem.

Theorem 3.22. Let $(X, \rho)$ be a D-metric space and let $f$ be a self-map on $X$. Suppose that there exists an $x_{0} \in X$ such that $O_{f}\left(x_{0}\right)=\left\{x_{0}\right\} \cup\left\{f^{n} x_{0}: n \in \mathbb{N}\right\}$ is bounded and every Cauchy sequence in $X$ converges to an element of $X$. Suppose also that

$$
\rho(f x, f y, f z) \leq \alpha \max \{\rho(x, y, z), \rho(x, f x, z)\}
$$

for $x, y, z \in \overline{O_{f}\left(x_{0}\right)}$, for some $\alpha \in[0,1)$. Then $f$ has a unique fixed point in $X$.

Remark 3.23. Example 3.7 shows that Theorem 3.22 is false. It also shows that [3, Corollaries 3.2, 3.3, 3.4, and 3.5] of Dhage et al. are false. In proving Theorem 3.22, the authors tacitly assume that the $D$-metric is sequentially continuous in two variables. With this additional assumption, the theorem remains true.

The following two theorems are generalizations of the modification of Theorem 3.22 suggested above.

Theorem 3.24. Let $(X, \rho)$ be a $D$ - metric space, let $f$ be a self-map on $X$, and let $\varphi: \mathbb{R}^{+} \rightarrow$ $\mathbb{R}^{+}$be a monotonically increasing map such that $\varphi(t+)<t$ for all $t \in(0, \infty)$. Suppose that there exists $x_{0} \in X$ such that $\sup \left\{\rho\left(x_{0}, f^{i} x_{0}, f^{j} x_{0}\right): i, j \in \mathbb{N} \cup\{0\}\right\}<+\infty$ and

$$
\rho(f x, f y, f z) \leq \varphi(\max \{\rho(x, u, v): u, v \in\{x\} \cup\{f x\} \cup\{y\} \cup\{z\}\})
$$

for all $x, y, z \in \overline{O_{f}\left(x_{0}\right)}$. Then $\left\{f^{n} x_{0}\right\}$ is a $\rho$-Cauchy sequence. If it converges to an element $p$ of $X$ and $\rho$ is sequentially continuous in any two variables, then $p$ is the unique fixed point of $f$ in $\overline{O_{f}\left(x_{0}\right)}$. 
Proof. Let $x_{n}=f^{n} x_{0}$ for all $n \in \mathbb{N}$.

For nonnegative integers $r$ and $s$, and $n \in \mathbb{N}$, from inequality (3.61), we have

$$
\begin{aligned}
& \rho\left(f^{n} x_{0}, f^{n} x_{r}, f^{n} x_{s}\right) \\
& \quad \leq \varphi\left(\max \left\{\rho\left(f^{n-1} x_{0}, f^{n-1} x_{p}, f^{n-1} x_{q}\right): p, q \in\{0,1\} \cup\{r\} \cup\{s\}\right\}\right) \\
& \quad \leq \varphi^{2}\left(\max \left\{\rho\left(f^{n-2} x_{0}, f^{n-2} x_{i}, f^{n-2} x_{j}\right): i, j \in\{0,1\} \cup\{r\} \cup\{s\}\right\}\right)
\end{aligned}
$$

(from the monotonic increasing nature of $\varphi$ )

$$
\vdots
$$

$$
\leq \varphi^{n}\left(\max \left\{\rho\left(x_{0}, x_{i}, x_{j}\right): i, j \in\{0,1\} \cup\{r\} \cup\{s\}\right\}\right) .
$$

Since $\sup \left\{\rho\left(x_{0}, f^{i} x_{0}, f^{j} x_{0}\right): i, j \in \mathbb{N} \cup\{0\}\right\}<+\infty$, there exists a positive real number $M$ such that $\rho\left(x_{0}, f^{i} x_{0}, f^{j} x_{0}\right) \leq M$ for all $i, j \in \mathbb{N} \cup\{0\}$. Hence, $\rho\left(f^{n} x_{0}, f^{n} x_{r}, f^{n} x_{s}\right) \leq \varphi^{n}(M)$ for all $n \in \mathbb{N}$. Since $\varphi(t+)<t$ for all $t \in(0, \infty),\left\{\varphi^{n}(t)\right\}$ decreases to zero for each $t \in$ $\mathbb{R}^{+}$. Hence, $\left\{\varphi^{n}(M)\right\}$ decreases to zero. Hence given $\varepsilon>0$, there exists $N \in \mathbb{N}$ such that $\varphi^{n}(M)<\varepsilon$ for all $n \geq N$. Hence, $\rho\left(f^{n} x_{0}, f^{n} x_{r}, f^{n} x_{s}\right)<\varepsilon$ for all $n \geq N$ and $r, s \in \mathbb{N} \cup\{0\}$. Hence, $\left\{f^{n} x_{0}\right\}$ is a $\rho$-Cauchy sequence.

Suppose that $\left\{f^{n} x_{0}\right\}$ converges to an element $p$ of $X$ with respect to $\rho$. From inequality (3.61), we have

$$
\begin{aligned}
& \rho\left(f^{n} x_{0}, f^{n} x_{0}, f p\right) \\
& \quad \leq \varphi\left(\max \left\{\rho\left(f^{n-1} x_{0}, u, v\right): u, v \in\left\{f^{n-1} x_{0}\right\} \cup\left\{f^{n} x_{0}\right\} \cup\{p\}\right\}\right) .
\end{aligned}
$$

Since $\left\{f^{n} x_{0}\right\}$ is a $\rho$-Cauchy sequence and $\rho$ is sequentially continuous in any two variables and $\left\{f^{n} x_{0}\right\}$ converges to $p$, it follows that $\max \left\{\rho\left(f^{n-1} x_{0}, u, v\right): u, v \in\left\{f^{n-1} x_{0}\right\} \cup\right.$ $\left.\left\{f^{n} x_{0}\right\} \cup\{p\}\right\}$ converges to zero. Hence on taking limits on both sides of inequality (3.63) as $n \rightarrow \infty$, we obtain

$$
\rho(p, p, f p) \leq \varphi(0+)
$$

Since $\varphi(t+)<t$ for all $t \in(0, \infty)$, we have $\varphi(0+)=0$. Hence $\rho(p, p, f p)=0$. Hence $f p=p$.

Let $w \in \overline{O_{f}\left(x_{0}\right)}$ be a fixed point of $f$. On taking $x=y=p$ and $z=w$ in inequality (3.61), we have

$$
\rho(p, p, w) \leq \varphi(\max \{\rho(p, p, w), \rho(p, w, w)\}) .
$$

On taking $x=p$ and $y=z=w$ in inequality (3.61), we have

$$
\rho(p, w, w) \leq \varphi(\max \{\rho(p, p, w), \rho(p, w, w)\}) .
$$


From inequalities (3.65) and (3.66), we have

$$
\max \{\rho(p, p, w), \rho(p, w, w)\} \leq \varphi(\max \{\rho(p, p, w), \rho(p, w, w)\})
$$

Since $\varphi(t) \leq \varphi(t+)<t$ for all $t \in(0, \infty)$, we must have

$$
\max \{\rho(p, p, w), \rho(p, w, w)\}=0 .
$$

Hence $w=p$. Hence $p$ is the unique fixed point of $f$ in $\overline{O_{f}\left(x_{0}\right)}$.

Remark 3.25. For $x, y, z \in X$, let $\alpha(x, y, z)=\max \{\rho(x, u, v): u, v \in\{x\} \cup\{f x\} \cup\{y\} \cup$ $\{z\}\}$. Since $\rho$ is symmetric in all the three variables and $\varphi$ is a monotonically increasing function on $\mathbb{R}^{+}$, inequality (3.61) is equivalent to the inequality

$$
\rho(f x, f y, f z) \leq \varphi(\min \{\alpha(x, y, z), \alpha(y, z, x), \alpha(z, x, y)\}) .
$$

Theorem 3.26. Let $(X, \rho)$ be a D-metric space, let $f$ be a self-map on $X$, and let $\varphi: \mathbb{R}^{+} \rightarrow \mathbb{R}^{+}$ be a monotonically increasing map such that $\varphi(t+)<t$ for all $t \in(0, \infty)$. Suppose that there exists $x_{0} \in X$ such that $O_{f}\left(x_{0}\right)$ is $\rho$-bounded and

$$
\rho(f x, f y, f z) \leq \varphi(\max \{\rho(u, v, w): u, v, w \in\{x\} \cup\{f x\} \cup\{y\} \cup\{f y\} \cup\{z\} \cup\{f z\}\})
$$

for all $x, y, z \in \overline{O_{f}\left(x_{0}\right)}$. Then $\left\{f^{n} x_{0}\right\}$ is a $\rho$-Cauchy sequence. If it converges to an element $p$ of $X$ and $\rho$ is sequentially continuous in any two variables, then $p$ is the unique fixed point of $f$ in $\overline{O_{f}\left(x_{0}\right)}$.

Proof. Let $x_{n}=f^{n} x_{0}$ for all $n \in \mathbb{N}$.

For $n \in \mathbb{N} \cup\{0\}$, let $\beta_{n}=\sup \left\{\rho\left(x_{i}, x_{j}, x_{k}\right): i, j, k \geq n\right\}$. Clearly $0 \leq \beta_{n} \leq \beta_{n-1}$ for all $n \in \mathbb{N}$. Since $O_{f}\left(x_{0}\right)$ is $\rho$-bounded, $\beta_{0}<+\infty$.

For $n \in \mathbb{N}$ and $i, j, k \geq n$, from inequality (3.70), we have

$$
\begin{aligned}
\rho\left(x_{i}, x_{j}, x_{k}\right) & =\rho\left(f x_{i-1}, f x_{j-1}, f x_{k-1}\right) \\
& \leq \varphi\left(\operatorname { m a x } \left\{\rho(u, v, w): u, v, w \in\left\{x_{i-1}\right\} \cup\left\{x_{i}\right\}\right.\right. \\
& \left.\left.\cup\left\{x_{j-1}\right\} \cup\left\{x_{j}\right\} \cup\left\{x_{k-1}\right\} \cup\left\{x_{k}\right\}\right\}\right) \\
& \leq \varphi\left(\beta_{n-1}\right) \quad \text { (since } \varphi \text { is monotonically increasing). }
\end{aligned}
$$

Hence, $\beta_{n} \leq \varphi\left(\beta_{n-1}\right)$ for all $n \in \mathbb{N}$. 
Hence, $\beta_{n} \leq \varphi^{n}\left(\beta_{0}\right)$ for all $n \in \mathbb{N}$. Since $\varphi(t+)<t$ for all $t \in(0, \infty)$ and $\beta_{0}$ is finite, $\varphi^{n}\left(\beta_{0}\right) \rightarrow 0$ as $n \rightarrow \infty$.

Hence, $\left\{\beta_{n}\right\}$ converges to zero. Hence, $\left\{x_{n}\right\}$ is a $\rho$-Cauchy sequence.

Suppose that $\left\{x_{n}\right\}$ converges to an element $p$ of $X$ and that the $D$-metric $\rho$ is sequentially continuous in any two variables.

For $n \in \mathbb{N}$, from inequality (3.70), we have

$$
\rho\left(x_{n}, x_{n}, f p\right) \leq \varphi\left(\max \left\{\rho(u, v, w): u, v, w \in\left\{x_{n-1}\right\} \cup\left\{x_{n}\right\} \cup\{p\} \cup\{f p\}\right\}\right) .
$$

Since $\left\{x_{n}\right\}$ is a $\rho$-Cauchy sequence, $\rho$ is sequentially continuous in any two variables and $\left\{x_{n}\right\}$ converges to an element $p$ of $X$, it follows that $\max \left\{\rho(u, v, w): u, v, w \in\left\{x_{n-1}\right\} \cup\right.$ $\left.\left\{x_{n}\right\} \cup\{p\} \cup\{f p\}\right\}$ converges to

$$
\max \{\rho(p, p, f p), \rho(p, f p, f p)\}
$$

On taking limits on both sides of inequality (3.72) as $n \rightarrow \infty$, we obtain

$$
\rho(p, p, f p) \leq \varphi((\max \{\rho(p, p, f p), \rho(p, f p, f p)\})+)
$$

In a similar manner, by taking $x=x_{n}, y=z=p$ in inequality (3.70) and then taking limits as $n \rightarrow \infty$, we obtain

$$
\rho(p, f p, f p) \leq \varphi((\max \{\rho(p, p, f p), \rho(p, f p, f p)\})+) .
$$

From inequalities (3.74) and (3.75), it follows that

$$
\begin{aligned}
\max & \{\rho(p, p, f p), \rho(p, f p, f p)\} \\
& \leq \varphi((\max \{\rho(p, p, f p), \rho(p, f p, f p)\})+) .
\end{aligned}
$$

Since $\varphi(t+)<t$ for all $t \in(0, \infty)$, we have

$$
\max \{\rho(p, p, f p), \rho(p, f p, f p)\}=0
$$

Hence $f p=p$. That $p$ is the only fixed point of $f$ in $\overline{O_{f}\left(x_{0}\right)}$ can be proved as in Theorem 3.24.

Remark 3.27. Let $(X, \rho)$ be as in Example 3.18. A sequence $\left\{x_{n}\right\}$ in $X$ converges to an element $x$ of $X$ with respect to $\rho$ if and only if $\left\{x_{n}\right\}$ converges to $x$ in the usual sense. Hence, it is evident that the $D$-metric $\rho$ is sequentially continuous in all the three variables. Hence, Example 3.18 illustrates Theorems 3.24 and 3.26 and the modified version of Theorem 3.22.

Definition 3.28. $f$ is said to be sequentially continuous at $x_{0} \in X$ if $\left\{f x_{n}\right\}$ converges to $f x_{0}$ whenever $\left\{x_{n}\right\}$ is a convergent sequence in $X$ with $x_{0}$ as a limit. $f$ is said to be sequentially continuous if it is sequentially continuous at every point of $X$. 
Theorem 3.29. Let $(X, \rho)$ be a D-metric space, let $f$ be a self-map on $X$, and let $\varphi: \mathbb{R}^{+} \rightarrow \mathbb{R}^{+}$ be a monotonically increasing map such that $\left\{\varphi^{n}(t)\right\}_{n=1}^{\infty}$ converges to zero for all $t$ in $\mathbb{R}^{+}$. Suppose that there exists $x_{0} \in X$ such that $O_{f}\left(x_{0}\right)$ is $\rho$-bounded and

$$
\rho(f x, f y, f z) \leq \varphi\left(\sup \left\{\rho(u, v, w): u, v, w \in O_{f}(x) \cup O_{f}(y) \cup O_{f}(z)\right\}\right)
$$

for all $x, y, z \in O_{f}\left(x_{0}\right)$. Then $\left\{f^{n} x_{0}\right\}$ is a $\rho$-Cauchy sequence. Suppose that $\left\{f^{n} x_{0}\right\}$ is convergent and has a unique limit and that $f$ is sequentially continuous at it. Then $f$ has a fixed point in $\overline{\mathrm{O}_{f}\left(x_{0}\right)}$.

Proof. For $n \in \mathbb{N}$, let $x_{n}=f^{n} x_{0}$.

For $n \in \mathbb{N} \cup\{0\}$, let $\beta_{n}=\sup \left\{\rho\left(x_{i}, x_{j}, x_{k}\right): i, j, k \geq n\right\}$. Clearly $0 \leq \beta_{n} \leq \beta_{n-1}$ for all $n \in \mathbb{N}$. Since $O_{f}\left(x_{0}\right)$ is $\rho$-bounded, $\beta_{0}<+\infty$.

For $n \in \mathbb{N}$ and integers $i, j, k \geq n$, from inequality (3.78), we have

$$
\begin{aligned}
& \rho\left(x_{i}, x_{j}, x_{k}\right) \\
& \quad \leq \varphi\left(\sup \left\{\rho(u, v, w): u, v, w \in O_{f}\left(x_{i}-{ }_{1}\right) \cup O_{f}\left(x_{j}-1\right) \cup O_{f}\left(x_{k}-{ }_{1}\right)\right\}\right) \\
& \quad \leq \varphi\left(\sup \left\{\rho\left(x_{r}, x_{s}, x_{t}\right): r, s, t \geq n-1\right\}\right) \quad\left(\text { since } \varphi \text { is monotonically increasing on } \mathbb{R}^{+}\right) \\
& \quad=\varphi\left(\beta_{n-1}\right) .
\end{aligned}
$$

Hence, $\beta_{n} \leq \varphi\left(\beta_{n-1}\right)$. Hence, $\beta_{n} \leq \varphi^{n}\left(\beta_{0}\right)$. Since $\left\{\varphi^{n}(t)\right\}_{n=1}^{\infty}$ converges to zero for all $t$ in $\mathbb{R}^{+}$, it follows that $\left\{\beta_{n}\right\}$ converges to zero. Hence, $\left\{x_{n}\right\}$ is a $\rho$-Cauchy sequence.

Suppose that $\left\{x_{n}\right\}$ is convergent and has a unique limit, say, $z$ in $X$ and that $f$ is sequentially continuous at $z$.

Since $\left\{f^{n} x_{0}\right\}$ converges to $z$ and $f$ is sequentially continuous at $z,\left\{f\left(f^{n} x_{0}\right)\right\}$ converges to $f z$. Hence, $f z$ is a limit of $\left\{x_{n}\right\}$.

Since $z$ is the only limit of $\left\{x_{n}\right\}$, we must have $f z=z$.

Remark 3.30. If $(X, \rho)$ is a $D$-metric space, $f$ is a self-map on $X, \varphi: \mathbb{R}^{+} \rightarrow \mathbb{R}^{+}$is continuous at 0 , and $\varphi(0)=0$ and $\rho(f x, f y, f z) \leq \varphi(\rho(x, y, z))$ for all $x, y, z \in X$, then $f$ is sequentially continuous.

Remark 3.31. Example 3.18 illustrates Theorem 3.29 also.

\section{References}

[1] B. Ahmad, M. Ashraf, and B. E. Rhoades, Fixed point theorems for expansive mappings in Dmetric spaces, Indian J. Pure Appl. Math. 32 (2001), no. 10, 1513-1518.

[2] B. C. Dhage, Generalised metric spaces and mappings with fixed point, Bull. Calcutta Math. Soc. 84 (1992), no. 4, 329-336.

[3] B. C. Dhage, A. M. Pathan, and B. E. Rhoades, A general existence principle for fixed point theorems in D-metric spaces, Int. J. Math. Math. Sci. 23 (2000), no. 7, 441-448.

[4] B. C. Dhage and B. E. Rhoades, A comparison of two contraction principles, Math. Sci. Res. HotLine 3 (1999), no. 8, 49-53.

[5] On common fixed points of quasi-contraction mappings in D-metric spaces, Indian J. Pure Appl. Math. 33 (2002), no. 5, 677-690. 
[6] G. Jungck, Common fixed points for noncontinuous nonself maps on nonmetric spaces, Far East J. Math. Sci. 4 (1996), no. 2, 199-215.

[7] S. V. R. Naidu, K. P. R. Rao, and N. Srinivasa Rao, On the topology of D-metric spaces and generation of D-metric spaces from metric spaces, Int. J. Math. Math. Sci. 2004 (2004), no. 51, 2719-2740.

S. V. R. Naidu: Department of Applied Mathematics, College of Science and Technology, Andhra University, Visakhapatnam-530 003, Andhra Pradesh, India

E-mail address: svrnaidu@hotmail.com

K. P. R. Rao: Department of Applied Mathematics, Acharya Nagarjuna University Post Graduate (ANUPG) Centre, Andhra Pradesh University, Nuzvid-521 201, India

E-mail address: kprrao2004@yahoo.com

N. Srinivasa Rao: Department of Applied Mathematics, Acharya Nagarjuna University Post Graduate (ANUPG) Centre, Andhra Pradesh University, Nuzvid-521 201, India

E-mail address: srinivasunimmala@yahoo.co.in 


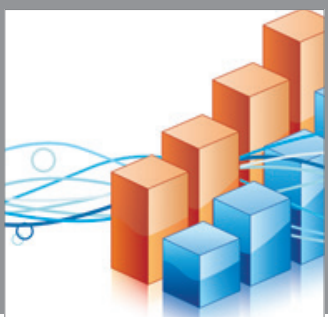

Advances in

Operations Research

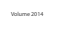

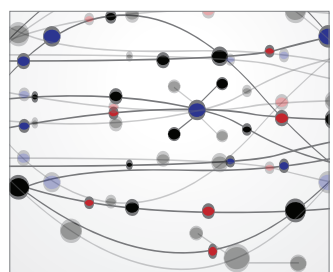

\section{The Scientific} World Journal
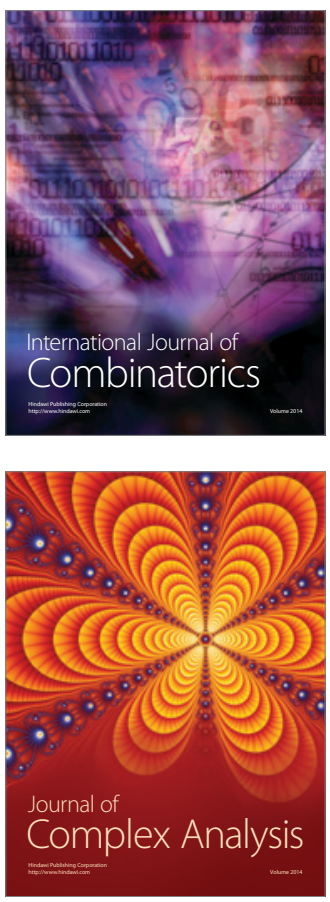

International Journal of

Mathematics and

Mathematical

Sciences
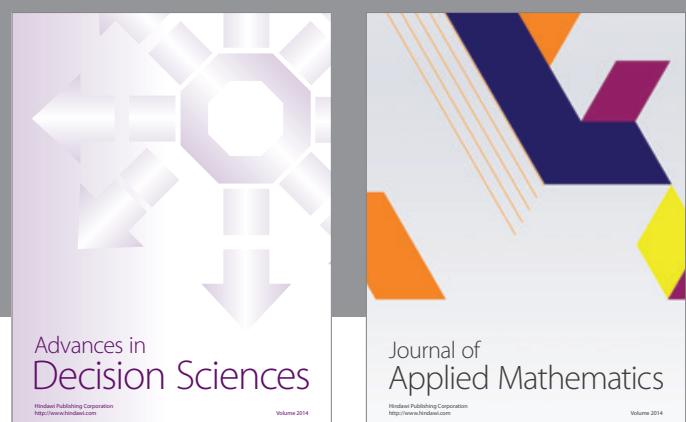

Journal of

Applied Mathematics
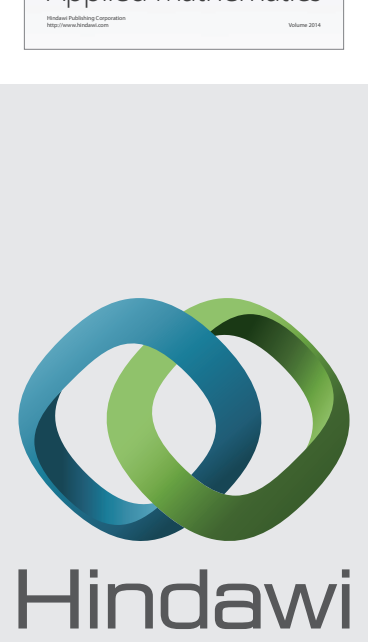

Submit your manuscripts at http://www.hindawi.com
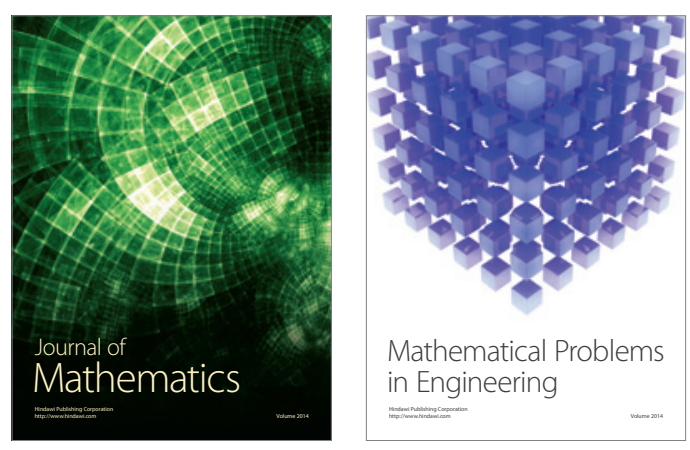

Mathematical Problems in Engineering
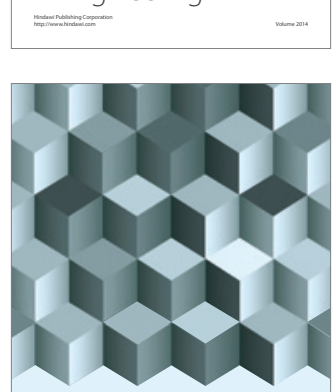

Journal of

Function Spaces
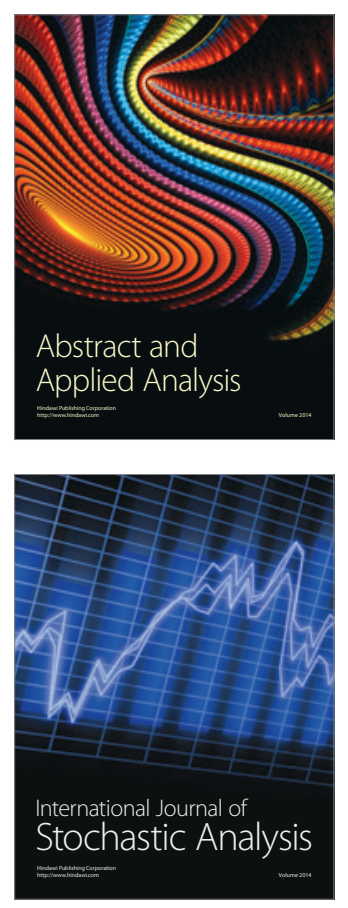

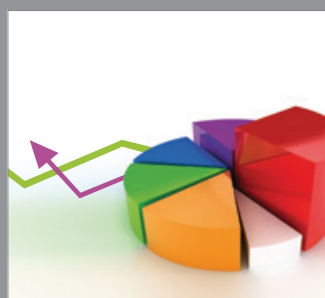

ournal of

Probability and Statistics

Promensencen
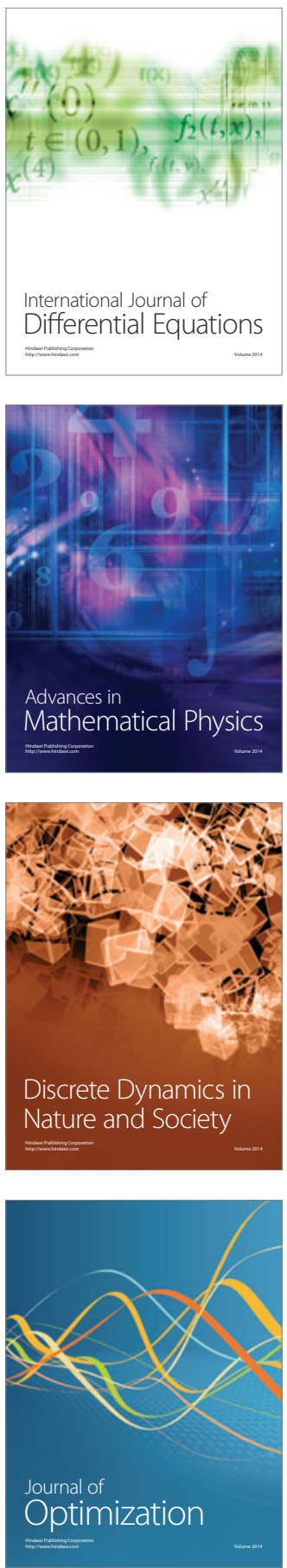